\title{
A Lagrangian-Eulerian model of particle dispersion in a turbulent plane mixing layer
}

\author{
L. A. Oliveira ${ }^{1, * \dagger}$, V. A. F. Costa ${ }^{2}$ and B. R. Baliga ${ }^{3}$ \\ ${ }^{1}$ Dep. de Eng. Mecânica (FCTUC-Polo II), Universidade de Coimbra, 3030-201 Coimbra, Portugal \\ ${ }^{2}$ Dep. de Eng. Mecânica, Universidade de Aveiro, Campus Universitário de Santiago, 3810-193 Aveiro, Portugal \\ ${ }^{3}$ Department of Mechanical Engineering, McGill University, 817 Sherbrooke St. West Montreal, \\ Quebec Canada H3A 2 K6
}

\begin{abstract}
SUMMARY
A Lagrangian-Eulerian model for the dispersion of solid particles in a two-dimensional, incompressible, turbulent flow is reported and validated. Prediction of the continuous phase is done by solving an Eulerian model using a control-volume finite element method (CVFEM). A Lagrangian model is also applied, using a Runge-Kutta method to obtain the particle trajectories. The effect of fluid turbulence upon particle dispersion is taken into consideration through a simple stochastic approach. Validation tests are performed by comparing predictions for both phases in a particle-laden, plane mixing layer airflow with corresponding measurements formerly reported by other authors. Even though some limitations are detected in the calculation of particle dispersion, on the whole the validation results are rather successful. Copyright (C) 2002 John Wiley \& Sons, Ltd.
\end{abstract}

KEY WORDS: multiphase flow; particle dispersion; Lagrangian-Eulerian; stochastic approach;

CVFEM; CFD

\section{INTRODUCTION}

Computational fluid dynamics (CFD) for two-phase flows is being increasingly applied in practice as an efficient, accessible and affordable way of supporting the design and optimization of industrial processes. Relevant applications include sprays, cyclones, pneumatic transport, particle-laden emissions of industrial effluents, particle or droplet combustion in industrial furnaces or energy converters, sediment transport and aerosol deposition, erosion, surface treatment and development of new materials, safety and fire suppression, food industry, among others (see References $[1,2]$ ). The geometrical configurations of practical interest are frequently very complex and may strongly affect the two-phase flow structure. Relatively *Correspondence to: L. A. Oliveira, Department de Eng. Mecânica (FCTUC-Polo II), Universidade de Coimbra,
3030-201 Coimbra, Portugal.

†E-mail: luis.adriano@dem.uc.pt

Contract/grant sponsor: Fundação para a Ciência e a Tecnologia; Contract/grant number: PECS/C/SAU/206/95

Received May 2001

Copyright (c) 2002 John Wiley \& Sons, Ltd.

Revised March 2002 
simple, versatile and reliable mathematical models that can be run by fast and robust computational codes are thus becoming an increasingly indispensable engineering design tool. As a contribution in this direction, a calculation procedure was recently developed by the present authors [3] for the two-dimensional transport of particles in the flow of a carrier fluid. A Lagrangian-type approach is used in the model to calculate both particle velocities and trajectories. Predictions for the continuous phase are performed by using a control-volume finite element method (CVFEM), thus providing the basis for easy inclusion of complex geometries in the calculation domain. The effect of fluid turbulence upon particle dispersion is taken into consideration through a 'particle-eddy dispersion model' described by Gosman and Ioannides [4]. The influence of the dispersed phase on fluid momentum and turbulence modulation are considered as well. This two-way coupling effect is included by use of the PSI-Cell model of Crowe et al. [5]. Preliminary tests have yielded successful results, ranging from simple verifications and comparisons with available analytical results to the evaluation of the dependence of fluid pressure drop on concentration of the dispersed phase (two-way coupling effect).

In this work, the above-mentioned theoretical procedure is briefly recalled and then applied to the modelling of particle transport in a turbulent, plane mixing shear layer of airflow. Detailed measurements for these fundamental, two-phase flow conditions were reported by Ando et al. [6], Hishida et al. [7], Ishima et al. [8]. The same problem was used as a test case for the Sixth Workshop on Two-Phase Flow Predictions [9], and also to validate an alternative numerical procedure by Coimbra et al. [10], where a control volume-type method is used to represent the continuous phase. Our aim is to validate the present numerical proposal through systematic comparisons of the corresponding predictions with this reliable experimental information.

\section{THE NUMERICAL METHOD}

\subsection{Control volume finite element method (CVFEM) for the fluid phase}

All the partial differential equations governing the steady two-dimensional turbulent situations involving fluid flow and related phenomena occurring in problems of interest here can be seen as particular cases of the following general differential equation, written for a unit volume in the conservative form and in Cartesian co-ordinates, $x_{i}$, for $i$ ranging from 1 to 2 [11, 12]:

$$
\frac{\partial}{\partial x_{i}}\left(\rho u_{i} \phi-\Gamma_{\text {eff, } \phi} \frac{\partial \phi}{\partial x_{i}}\right)=S_{\phi}
$$

where the Einstein convention is adopted for the index $i$. In Equation (1), where the first term inside the brackets accounts for convection, $\phi$ is a general dependent variable, to which are associated specific values or expressions for the corresponding effective diffusion coefficient, $\Gamma_{\text {eff, } \phi}$ and source term, $S_{\phi}$. As listed in Table I, the variable $\phi$ may be interpreted as a velocity component $u_{i}$ or as unity, in which case Equation (1) represents momentum or mass conservation, respectively. Turbulent transport is simulated by the standard high-Reynolds number $k-\varepsilon$ turbulence model of Launder and Spalding [13], in which Equation (1) must be solved for two additional variables: the turbulence kinetic energy, $k=\overline{u_{i}^{\prime} u_{i}^{\prime}} / 2$, and its rate of dissipation $\varepsilon=(\mu / \rho) \overline{\left(\partial u_{i}^{\prime} / \partial x_{j}\right)^{2}}$, where $u_{i}^{\prime}$ is the fluctuating part of velocity component, $u_{i}$, and $\rho$ and $\mu$ stand for the fluid density and dynamic viscosity, respectively. In this work, $\rho$ and $\mu$ 
Table I. Values of $\Gamma_{\text {eff, } \phi}$ and $S_{\phi}$ in general transport Equation (1).

\begin{tabular}{lcc}
\hline$\phi$ & $\Gamma_{\text {eff }, \phi}$ & $S_{\phi}$ \\
1 & 0 & 0 \\
$u_{i}$ & $\mu+\mu^{\mathrm{t}}$ & $-\frac{\partial p}{\partial x_{i}}+S_{u_{i}}^{\mathrm{p}}$ \\
$k$ & $\mu+\mu^{\mathrm{t}} / \sigma_{k}$ & $P_{k}-\rho \varepsilon+S_{k}^{\mathrm{p}}$ \\
$\varepsilon$ & $\mu+\mu^{\mathrm{t}} / \sigma_{\varepsilon}$ & $\left(C_{1 \varepsilon} P_{k}-C_{2 \varepsilon} \rho \varepsilon\right) \frac{\varepsilon}{k}+S_{\varepsilon}^{\mathrm{p}}$ \\
\hline
\end{tabular}

Table II. Values assigned to the empirical constants in the $k-\varepsilon$ model.

\begin{tabular}{lcccc}
\hline$C_{\mu}$ & $\sigma_{k}$ & $\sigma_{\varepsilon}$ & $C_{1 \varepsilon}$ & $C_{2 \varepsilon}$ \\
0.09 & 1.0 & 1.3 & 1.44 & 1.92 \\
\hline
\end{tabular}

are assumed to remain constant. The 'turbulent viscosity', $\mu^{\mathrm{t}}$, and the energy production term, $P_{k}$, appearing in Table I, are defined as $\mu^{\mathrm{t}}=C_{\mu} \rho k^{2} / \varepsilon$ and $P_{k}=\mu^{\mathrm{t}}\left(\partial u_{i} / \partial x_{j}+\partial u_{j} / \partial x_{i}\right) \partial u_{i} / \partial x_{j}$, respectively ( $j$ also ranging from 1 to 2 ). The source contributions from the particle phase, $S_{u_{i}}^{\mathrm{p}}, S_{k}^{\mathrm{p}}$ and $S_{\varepsilon}^{\mathrm{p}}$, are defined in Equations (12), (13a) and (13b), respectively. The values assigned to the empirical constants of the model, $C_{\mu}, \sigma_{k}, \sigma_{\varepsilon}, C_{1 \varepsilon}$ and $C_{2 \varepsilon}$ are specified in accordance with Reference [13], as listed in Table II.

The calculations are not explicitly carried out all the way to solid, impermeable walls. Rather, the wall function treatment proposed by Launder and Spalding [13] is adopted. Thus, the mean velocity component parallel to the wall and the distance normal to the wall are nondimensionalized as follows: $u^{+}=u / u_{\tau}$ and $y^{+}=y u_{\tau} /(\mu / \rho)$, where $u_{\tau}=\sqrt{\tau_{w} / \rho}$ is the friction velocity and with $\tau_{w}$ denoting the shear stress at the wall. In the regions adjacent to solid, impermeable walls, it is assumed that for $y^{+} \geqslant 11.2, u^{+}=\ln \left(E y^{+}\right) / \chi$, with $E=9.8$ for smooth walls, and $\chi=4.2$ is the von Kármán constant; for $y^{+}<11.2$, the approximation $u^{+}=y^{+}$is used. Furthermore, in the near-wall region, it is assumed that the production and dissipation rates of turbulent kinetic energy are in equilibrium. After some algebraic manipulations [14], this simplified model leads to the following relations for $k$ and $\varepsilon$, respectively, which were used at near-wall (nw) grid points in the calculation domain:

$$
k_{\mathrm{nw}}=\frac{1}{\sqrt{C_{\mu}}}\left[\frac{\chi}{\ln \left(E y^{+}\right)}\right]^{2} u^{2}, \quad \varepsilon_{\mathrm{nw}}=\frac{\left(C_{\mu} k^{2}\right)^{3 / 4}}{\chi y}
$$

where $C_{\mu}=0.09$ is one of the five empirical constants recommended by Launder and Spalding. With this wall-function treatment, at near-wall grid points with $y^{+} \geqslant 11.2$,

$$
\tau_{\mathrm{nw}}=\frac{\chi \rho \sqrt[4]{C_{\mu} k^{2}}}{\ln \left(E y^{+}\right)} u
$$

while for grid points with $y^{+}<11.2, \tau_{\mathrm{nw}}=\mu u / y$. Noting the constant wall shear stress assumption invoked in the wall-function treatment, these expressions for $\tau_{\mathrm{nw}}$, after being multiplied by the appropriate area, are the boundary conditions that were used in momentum balances over control volumes adjacent to solid, impermeable, walls. The wall-function approach is exclusively applied to the wall boundaries of the domain. 
For the present test case, to be described in Section 3, zero-normal-gradient conditions are assumed to hold at the outflow boundaries of the calculation domain [15], while inlet values of the dependent variables are set equal to experimental data.

Equation (1) is numerically integrated by use of the CVFEM, reviewed recently by Baliga [16], with inclusion of a mass-weighted (MAW) scheme [17, 18] for discretization of the convective terms. Geometric versatility is ensured by dividing the domain into three-node triangular elements, where curved boundaries are approximated by piecewise-linear segments. The control volumes are formed by joining the centroids of the elements to the midpoints of the corresponding sides, thus creating a polygonal control volume around each node throughout the calculation domain. Further details of the whole procedure can be found in Reference [11].

\subsection{Lagrangian tracking procedure for the particle phase}

The solid phase is here assumed to be composed of hard, spherical, non-rotating, smooth, elastic particles of uniform diameter $d^{\mathrm{p}}$, mass $m^{\mathrm{p}}$ and mass density $\rho^{\mathrm{p}}$, where the superscript, $\mathrm{p}$, is used to identify the particulate phase. In the problems considered here, the only significant forces acting on each particle are the drag, gravitation and local pressure gradient forces. The equation of motion of a single particle of unit mass is thus reduced to the following form [19]:

$$
\frac{\mathrm{d} u_{i}^{\mathrm{p}}}{\mathrm{d} t}=\frac{3}{4} \frac{\mu}{\rho^{\mathrm{p}}\left(\mathrm{d}^{\mathrm{p}}\right)^{2}} C_{D} R e^{\mathrm{p}}\left(u_{i}-u_{i}^{\mathrm{p}}\right)+g_{i}\left(1-\frac{\rho}{\rho^{\mathrm{p}}}\right)-\frac{1}{\rho^{\mathrm{p}}} \frac{\partial p}{\partial x_{i}}
$$

where the three terms of the right-hand side represent, in the same order, the forces that were mentioned above are responsible for the particle acceleration (left-hand side). In Equation (4) the particle Reynolds number is based upon the relative velocity $\left|\vec{V}^{\mathrm{rel}}\right|=\sqrt{\left(u_{i}-u_{i}^{\mathrm{p}}\right)^{2}}$ and its product with the drag coefficient $C_{\mathrm{D}}$ is calculated here using the following empirical relations [20]:

$$
C_{\mathrm{D}} R e^{\mathrm{p}}= \begin{cases}24+3.6\left(R e^{\mathrm{p}}\right)^{0.687} & \text { if } R e^{\mathrm{p}} \leqslant 1000 \\ 0.44 R e^{\mathrm{p}}, & \text { if } R e^{\mathrm{p}}>1000\end{cases}
$$

Particle trajectories, $x_{i}^{\mathrm{p}}$, are related to the instantaneous particle velocity by the following equation:

$$
\frac{\mathrm{d} x_{i}^{\mathrm{p}}}{\mathrm{d} t}=u_{i}^{\mathrm{p}}
$$

The integration of Equations (4) and (6) is here performed numerically through a fourth-order Runge-Kutta procedure. The particle position and velocity are thus calculated for a time level $t+\Delta t$ as functions of the corresponding values that are available for the previous time level $t$. Selection of the time step, $\Delta t$, is based on the local value of the Stokes number $S t=\tau^{\mathrm{p}} / \tau$, where $\tau^{\mathrm{p}}$ and $\tau$ are characteristic particle and fluid velocity response times, respectively. The expression for $\tau^{\mathrm{p}}$ is [21]:

$$
\tau^{\mathrm{p}}=-\tau^{\mathrm{s}} \ln \left[1-\frac{\left(1-e^{-1}\right) / \tau_{\mathrm{s}}}{1 / \tau_{\mathrm{s}}+K_{0}}\right]
$$


where $\tau^{\mathrm{s}}$ is the particle response time if only drag forces are considered to act upon the particle [21]:

$$
\tau^{\mathrm{s}}=\frac{4}{3} \frac{\rho^{\mathrm{p}}\left(d^{\mathrm{p}}\right)^{2}}{\mu}\left(C_{\mathrm{D}} R e^{\mathrm{p}}\right)^{-1}
$$

and $K_{0}=\sum\left|\vec{F} / m^{\mathrm{p}}\right| / \sqrt{u_{i}^{2}}$ is a correction factor that includes the remaining forces $\vec{F}$ (pressure and gravity, in the present case). The fluid response time, $\tau$, is defined as

$$
\tau=\frac{\delta}{\sqrt{u_{i}^{2}}}
$$

where $\delta$ is a characteristic dimension of the Eulerian grid (here taken as the square root of the area of the cell where the particle is localised at instant $t$ ). In practice, if $S t \ll 1$, the particle essentially follows the fluid flow, and the time step is set as $\Delta t=0.1 \tau$; if $S t \approx 1$ or $S t \gg 1$, then $\Delta t=0.1 \tau^{\mathrm{p}}$. Anyway, $\Delta t$ should never exceed the stability limit that accounts for the explicit nature of the Runge-Kutta method [22].

In Equation (4) the knowledge of the fluid forces that are responsible for particle motion requires the determination of the CVFEM mesh element in which the particle is located at each Lagrangian time level. This is performed by using geometrical arguments. Namely a particle position $\vec{P}$ lies inside a triangular element of vertices $1,2,3$ if the cross-products $\overrightarrow{12} \times \overrightarrow{1 P}, \overrightarrow{23} \times \overrightarrow{2 P}, \overrightarrow{31} \times \overrightarrow{3 P}$ are all positive or all negative. The local fluid velocity and pressure gradient are then calculated by linear interpolation of the corresponding values already available at the grid nodes 1,2 and 3. Geometrical reasoning is also used to determine the residence time, or time spent by the particle in each triangular element it crosses. This is necessary for an averaging procedure and also to account for two-way coupling effects, as will be seen later. The presence of solid boundaries is modelled through elastic collisions with introduction of a restitution coefficient. The trajectory calculation is terminated when the particle leaves the calculation domain at outflow boundaries.

The effect of fluid turbulence upon particle dispersion is based on the concept of particleeddy encounters (cf. Reference [4]). The instantaneous fluid velocity components to be used in Equation (4) are estimated by superimposing, on the corresponding mean values, fluctuating components $u_{i}^{\prime}$ that are obtained by randomly sampling a Gaussian distribution with standard deviation $(2 k / 3)^{0.5}$ :

$$
u_{i}^{\prime}=\sqrt{2 k / 3} \zeta_{i}
$$

where $\zeta_{i}(i=1,2)$ are two independent random variables with zero mean and unit variance. The time duration of particle-eddy interaction is estimated as the minimum of two time scales: an eddy transit time and an eddy lifetime. This interaction time should never be exceeded by the Lagrangian time step $\Delta t$ used in the determination of the particle trajectory. The instantaneous fluid velocities are updated every time the particle encounters a new eddy.

\subsection{Eulerian-Lagrangian modelling of the particle-laden flow}

Two-way coupling effects are included in the present model through the particle source-in cell (PSI-CELL) strategy of Crowe et al. [5]. The global calculation procedure may be described as follows: First the fluid flow is predicted ignoring the presence of particles; these fluid flow 
results are then frozen and all particle trajectories calculated, thus leading to the determination of momentum and turbulent energy source (or sink) contributions for each control volume that has been visited by the particles along their paths; with these new source terms, the CVFEM calculations are redone for the fluid flow; then the former particle trajectories are corrected. The whole procedure is repeated until convergence is achieved.

Along its own trajectory, a particle with mass $m^{\mathrm{p}}$ is representative of a number of analogous particles introduced in the domain at a rate $\dot{N}$ through the same injection point. Each trajectory $j$ is thus associated with a particle mass flow $\dot{m}_{j}^{\mathrm{p}}=\dot{N}_{j} m^{\mathrm{p}}$. As it crosses an Eulerian cell of volume $\Delta v$, a momentum source contribution is generated for the continuous phase $[5,23]$ :

$$
\left(S_{u_{i}}^{\mathrm{p}}\right)_{j} \Delta v=-\dot{m}_{j}^{\mathrm{p}}\left[\left(u_{i}^{\mathrm{p}}\right)_{\text {out }}-\left(u_{i}^{\mathrm{p}}\right)_{\text {in }}\right]+\dot{m}_{j}^{\mathrm{p}}\left(t_{\text {out }}-t_{\text {in }}\right)\left[g_{i}\left(1-\frac{\rho}{\rho^{p}}\right)-\frac{1}{\rho^{p}} \frac{\partial p}{\partial x_{i}}\right]
$$

Equation (11) shows that the particle momentum source term is composed of two parts: the change of momentum of the particles (net efflux of particle momentum), and the influence of the external forces (gravity and local pressure gradient) acting on the particles. In this equation, $g_{i}$ is the gravity acceleration along direction $i$, and the indices in and out refer to the particle entering and leaving the control volume, respectively. If that control volume is traversed by a number of trajectories, $J$, the resulting source contribution from the particle phase to the source term $S_{u_{i}}$ in Equation (1) (with $\phi \equiv u_{i}$ ) becomes:

$$
S_{u_{i}}^{\mathrm{p}} \Delta v=\sum_{j=1}^{J}\left(S_{u_{i}}^{\mathrm{p}}\right)_{j}
$$

Analogously, and following Gouesbet et al. [24], the influence of particles upon the turbulence level of the carrier phase (turbulence modulation) is accounted for through source term contributions to be included in Equation (1) (with $\phi \equiv k, \varepsilon$ ):

$$
\begin{aligned}
S_{k}^{\mathrm{p}} \Delta v & =\overline{u_{i} S_{u_{i}}^{p}}-\overline{u_{i}} \overline{S_{u_{i}}^{p}} \\
S_{\varepsilon}^{\mathrm{p}} \Delta v & =1.1 \frac{\varepsilon}{k} S_{k}^{\mathrm{p}}
\end{aligned}
$$

where the time averages are computed on all the trajectories crossing the Eulerian control volume. In each Eulerian cell crossed by a number $J$ of particle trajectories, a particle volume concentration, $\alpha$, is defined by

$$
\alpha=\frac{\sum_{j=1}^{J} \dot{N}_{j}\left(t_{\text {out }}-t_{\text {in }}\right)_{j} \pi\left(d^{\mathrm{p}}\right)^{3} / 6}{\Delta v}
$$

where $\dot{N}_{j}$ is the number of particles per unit time that cross the cell along trajectory $j$, and $\left(t_{\text {out }}-t_{\text {in }}\right)_{j}$ is the time that each of these particles takes to traverse the cell.

\section{RESULTS}

The test case adopted for validation of the present numerical procedure is the dispersion of spherical glass particles $\left(\rho^{\mathrm{p}}=2590 \mathrm{~kg} / \mathrm{m}^{3}\right)$ in a plane mixing layer formed by two air 


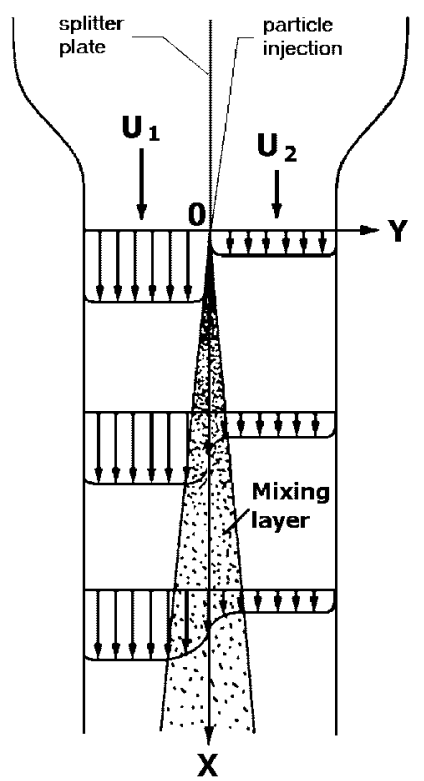

Figure 1. Flow configuration and reference frame.

streams having different uniform velocity profiles at the inlet. The test section $(450 \mathrm{~mm}$ long and $150 \times 100 \mathrm{~mm}^{2}$ in cross section) is installed vertically as represented in Figure 1, where a Cartesian co-ordinate system $\left(x_{1} \equiv x, x_{2} \equiv y\right)$ is also defined, together with the two corresponding components of the velocity field $\left(u_{1} \equiv u, u_{2} \equiv v\right)$. Particles of uniform diameter $d^{\mathrm{p}}$ ranging from 42 to $135 \mu \mathrm{m}$ (associated with mass flow rates between 7.5 and $20.43 \mathrm{~g} / \mathrm{s}$, respectively) are injected into the flow through a $y$-oriented slit of width $\Delta y=0.57 \mathrm{~mm}$, existing in the splitter plate that is used to separate the inlet air streams of uniform velocities $U_{1}=13.0 \mathrm{~m} / \mathrm{s}, U_{2}=4.0 \mathrm{~m} / \mathrm{s}$ and turbulence intensities $0.9,0.2 \%$, respectively. Laboratory experiments for these conditions were conducted by Ando et al. [6], Hishida et al. [7] and Ishima et al. [8]. The same configuration was adopted as a test case for the Sixth Workshop on Two-Phase Flow Predictions [9]. The experimental information in References [6, 7] is used in this section as a comparative reference for the present numerical experiments. A detailed description and a schematic representation of the whole experimental set-up may be found in Reference [7]. With reference to Figure 1, the spatial domain used in the numerical predictions is defined by $(0 \leqslant x \leqslant 450 \mathrm{~mm},-50 \mathrm{~mm} \leqslant y \leqslant 50 \mathrm{~mm})$. The inflow boundary (for both the two air streams and the particles) is located at $x=0$. The $0.57 \mathrm{~mm}$ slit in the splitter plate used for particle injection is centred around the origin of the co-ordinate system (point $\mathrm{O}$, in the figure). The inclusion in Figure 1 of a small region where $x<0$ is only motivated to ensure clarity.

Approximate grid independence is ensured by using a non-uniform $(x, y)$ Eulerian mesh with $79 \times 99$ nodes, gradually compressed for decreasing values of $x$ and $|y-50|$. This is illustrated in Figure 2, where the streamwise distribution of the local momentum thickness, 


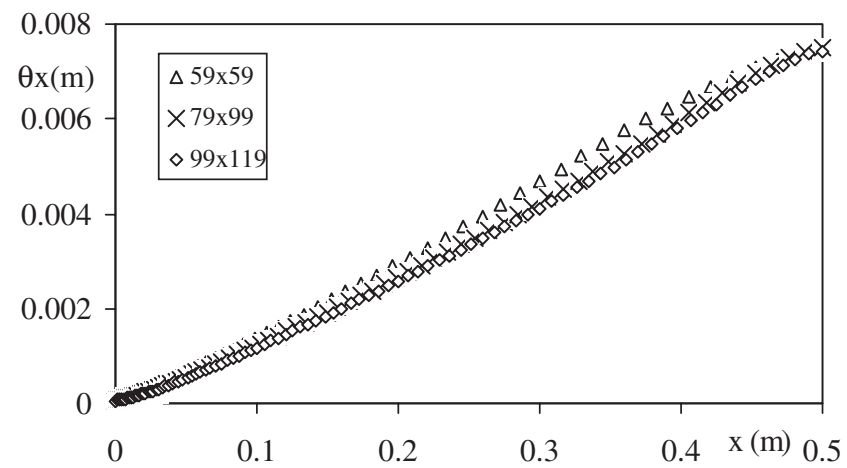

Figure 2. Streamwise distribution of the local momentum thickness, $\theta_{x}$, obtained for different computational grids.

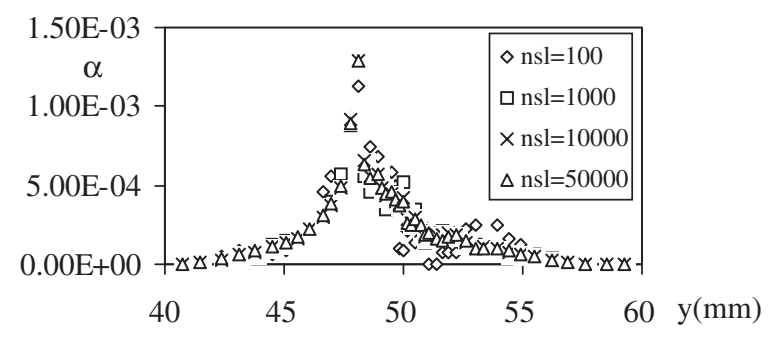

Figure 3. Distributions of particle volume concentration at a downstream distance $x=200 \mathrm{~mm}$, for different values of nsl. Particle diameter: $d^{\mathrm{p}}=135 \mu \mathrm{m}$.

defined as

$$
\theta_{x}=\int_{-\infty}^{+\infty} \frac{u-U_{2}}{\Delta U}\left(1-\frac{u-U_{2}}{\Delta U}\right) \mathrm{d} y
$$

is represented using non-uniform grids of $59 \times 59,79 \times 99$ and $99 \times 119$ nodes. In general, results obtained for the relevant variables through use of the last two grids showed no significant differences between them.

Starting points for particle trajectories are regularly distributed along the inlet slit. A large number of starting points, nsl, is necessary in order to guarantee that the predicted average properties of the particulate phase are nearly insensitive to the number of particle trajectories considered in the Lagrangian stochastic model. Sample calculations were performed using nsl equal to $10^{2}, 10^{3}, 10^{4}$ and $5 \times 10^{4}$ with no significant differences in the last two cases. An example is shown in Figure 3 where, for particles of $d^{\mathrm{p}}=135 \mu \mathrm{m}$, predicted profiles of particle volume concentration, $\alpha$, at a downstream distance $x=200 \mathrm{~mm}$ are represented for different numbers of starting points. On the basis of these numerical experiments, the value $\mathrm{nsl}=5 \times 10^{4}$ is thus adopted in all subsequent predictions. A similar value $(\mathrm{nsl}=49570)$ was used by Coimbra et al. [10] for the same purpose. Furthermore, the measurements reported by Hishida et al. [7] show that the gas velocity profiles with or without the presence of 

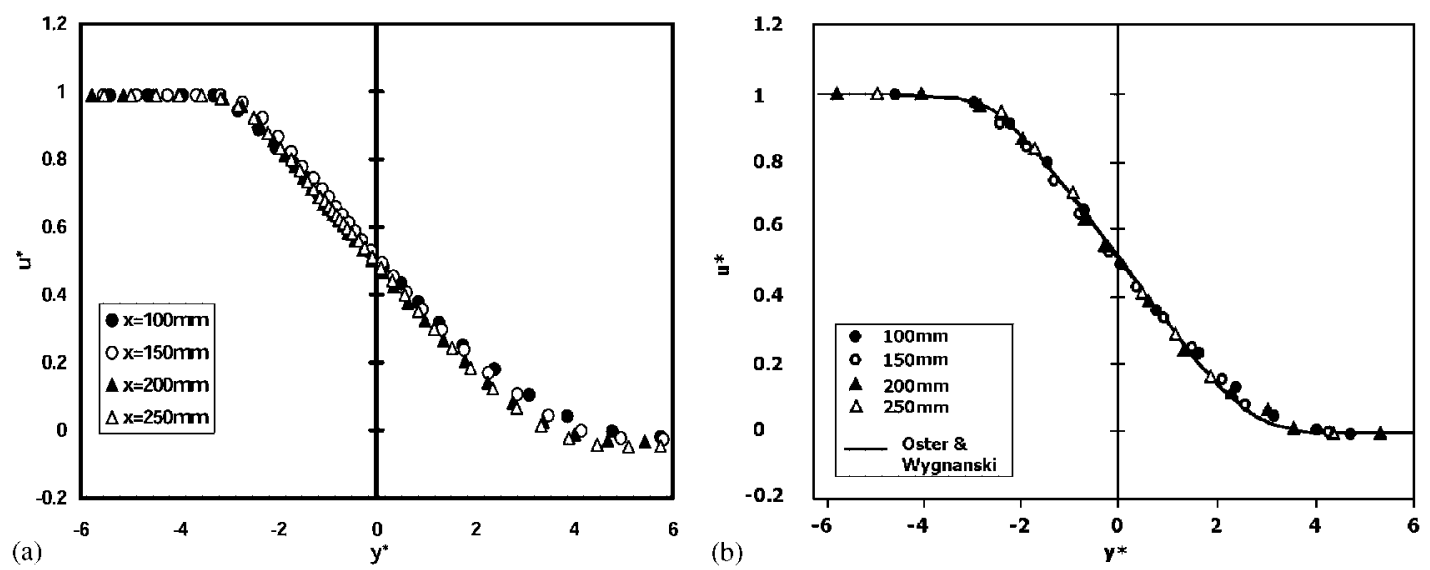

Figure 4. Single phase: streamwise mean velocity profiles for different longitudinal locations. (a) present predictions; (b) measurements reported by Hishida et al. [7].

particles differ by less than $3 \%$. Thus, one-way coupling calculations are adequate for the present purposes.

\subsection{Single phase flow results}

According to the measurements of Hishida et al. [7], the fluid mixing layer is expected to be fully developed for downstream locations over $x=100 \mathrm{~mm}$. Following those authors, the present predictions for the mean fluid velocity in the streamwise direction, $u$, are normalized as $u^{*}=\left(u-U_{2}\right) / \Delta U$ and plotted against a dimensionless transversal coordinate defined by $y^{*}=\left(y-y_{0.5}\right) / \theta_{x}$, where $\Delta U=U_{1}-U_{2}$ and $y_{0.5}$ is the $y$ location for which $u=0.5\left(U_{1}+U_{2}\right)$. This is shown for several streamwise locations in Figure 4, where profile similarity is in fact seen to hold. The present predictions are nearly coincident with those measurements, also shown for comparison (for clarity and within graphical precision, one single experimental curve may be taken as representative of the different longitudinal locations).

In Figure 5 the predicted streamwise dimensionless root mean square velocity profiles

$\left(u_{\mathrm{rms}}^{*}=\sqrt{\overline{\left(u^{\prime}\right)^{2}}} / \Delta U\right)$ are displayed for different longitudinal positions and compared with the corresponding measurements reported by Oster and Wygnanski [25] for a fully developed two-dimensional mixing layer. The maximum values of the predicted distributions increase with the downstream distance and tend to match the top value of the Gaussian-type experimental profile. The fluctuating level is underpredicted on the high velocity side, a trend that can also be observed in the numerical calculations of Coimbra et al. [10]. The reason for this discrepancy is that the present isotropic eddy-viscosity model cannot adequately account for the actual anisotropy of this turbulent flow. However, the overall agreement may be quite reasonable for engineering design considerations.

\subsection{Two phase flow results}

Figure 6 shows a comparison between predicted and measured transversal distributions of the average particle longitudinal velocities at the downstream location $x=200 \mathrm{~mm}$. According to 

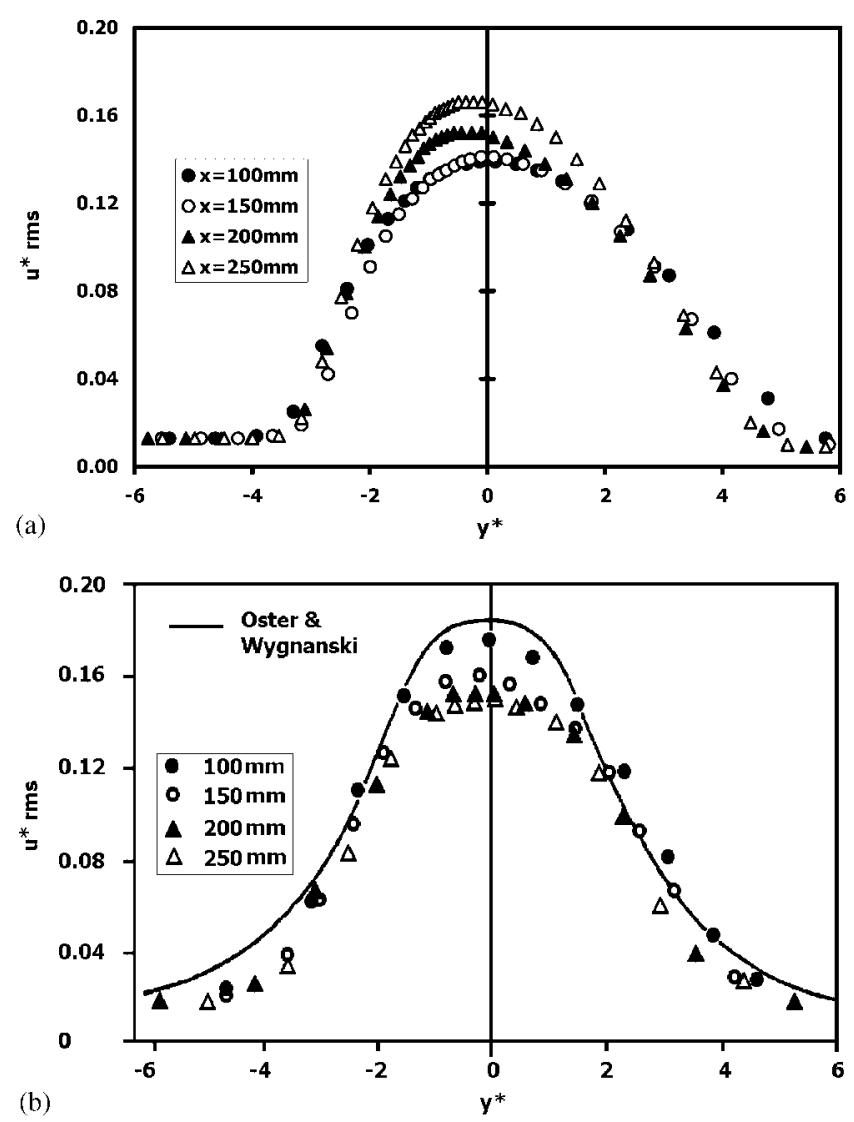

Figure 5. Single phase: streamwise rms velocity profiles for different longitudinal locations. (a) present predictions; (b) measurements reported by Hishida et al. [7].

the experiments, particle diameters vary from 42 to $135 \mu \mathrm{m}$, with an intermediate value of $d^{\mathrm{p}}=72 \mu \mathrm{m}$. The corresponding profile of the fluid phase is also represented for reference.

Particles with the smallest response time $\left(d^{\mathrm{p}}=42 \mu \mathrm{m}, \tau^{\mathrm{p}}=0.014 \mathrm{~s}\right)$ nearly follow the gas phase, while the largest ones $\left(d^{\mathrm{p}}=135 \mu \mathrm{m}, \tau^{\mathrm{p}}=0.144 \mathrm{~s}\right)$ are unable to reach the fluid velocity. This experimentally observed behaviour is seen to be reasonably well predicted by the present calculations. However, the experimental measurements show that the average velocity of particles with an intermediate diameter $\left(d^{\mathrm{p}}=72 \mu \mathrm{m}, \tau^{\mathrm{p}}=0.041 \mathrm{~s}\right)$ do exceed the fluid velocity in the lower velocity side. This trend is totally absent in the numerical predictions. Actually, such a disagreement should be expected, as the response time of those particles is of the same order of magnitude as the characteristic time of the fluid turbulence large scale eddies. Particles may thus be trapped within those eddies and then thrown away (in this case from the higher to the lower velocity side) due to their own inertia and keeping their initial average longitudinal momentum. The correct representation of this 'overshoot' phenomenon of particle dispersion would require the resolution of the time-dependent Navier-Stokes equations 

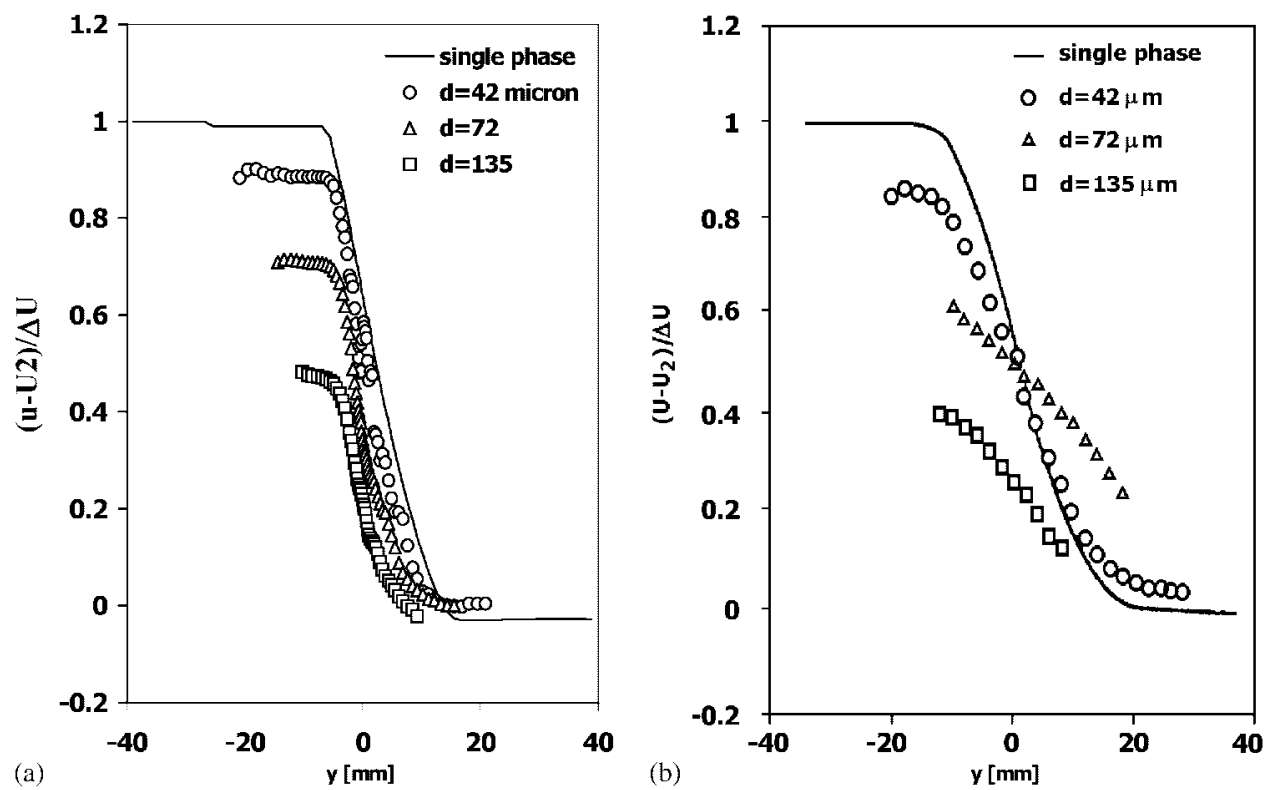

Figure 6. Particle phase: streamwise average velocity profiles at $x=200 \mathrm{~mm}$ for different particle diameters. (a) present predictions; (b) measurements reported by Hishida et al. [7]. The single phase normalization procedure is also adopted for the particulate phase velocities.

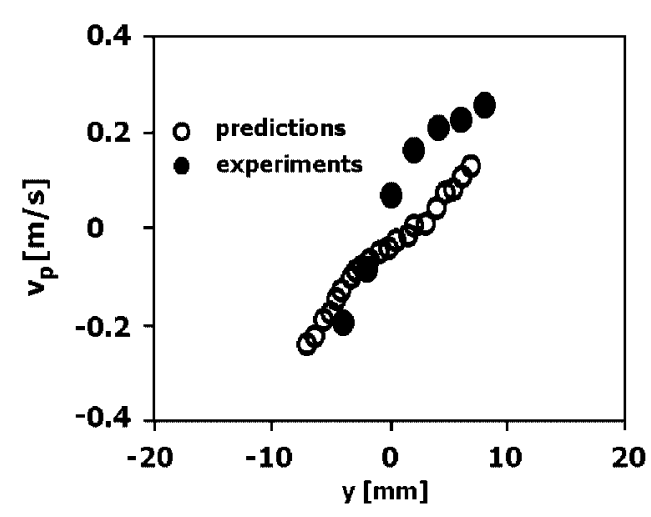

Figure 7. Particle phase, $d^{\mathrm{p}}=135 \mu \mathrm{m}$ : comparison between present predictions for the transverse average velocity profile at the downstream distance $x=100 \mathrm{~mm}$ and the corresponding measurements reported by Coimbra et al. [10].

for the gas phase, which is obviously beyond the possibilities of the present turbulent transport modelling.

A sampling comparison between the presently calculated distribution of the average transverse particle velocity component and the corresponding experimental profile obtained by Coimbra et al. [10] is established in Figure 7 for a cross section located at $x=100 \mathrm{~mm}$, for 


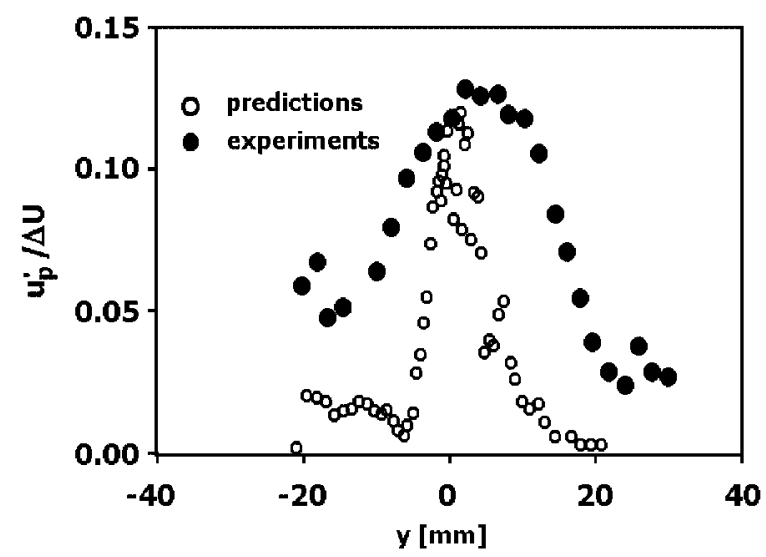

Figure 8. Particle phase, $d^{\mathrm{p}}=42 \mu \mathrm{m}$ : comparison between predicted and measured profiles of longitudinal velocity fluctuation (rms), at the downstream location $x=200 \mathrm{~mm}$.

$d^{\mathrm{p}}=135 \mu \mathrm{m}$. Even though there is global agreement, in the low velocity side, $v^{\mathrm{p}}$ is slightly underpredicted.

Taking as example the dispersion of $42 \mu \mathrm{m}$ diameter particles and a cross section located at $x=200 \mathrm{~mm}$, the calculated distributions of particle streamwise velocity fluctuations, $u^{\prime p}$, are shown in Figure 8 and compared with the corresponding experimental values of Hishida et al. [7]. As in the measurements, the predicted level of particle velocity fluctuation is considerably higher in the streamwise direction than in the transverse direction (not shown). However, in both cases the calculations underestimate the corresponding values. This may be seen as a consequence of the time-uncorrelated nature of the present representation for particle dispersion, together with the inability of the $k-\varepsilon$ turbulence model to account for the anisotropy of the gas phase shear flow itself. Particle long-time dispersions, particularly sensitive to such a combined limitation, are actually important in the present two-phase, turbulent mixing-layer type flow.

As should be expected, the tendency to underestimate particle velocity fluctuation is less noticeable for higher inertia particles. This can be seen from observation of Figure 9 where, for a common particle diameter $\left(d^{\mathrm{p}}=135 \mu \mathrm{m}\right)$, predicted and measured profiles of dimensionless particle number density $\alpha / \alpha_{\max }$ are compared at two downstream locations, namely $x=100 \mathrm{~mm}$ (a) and $x=200 \mathrm{~mm}$ (b). As in Hishida et al. [7], the vertical scale is here normalized by the maximum value $\alpha_{\max }$ at $x=100 \mathrm{~mm}$. Experimental evidence shows that increasing downstream distances correspond to a decay of the maximum value and also to a broadening effect of the particle average concentration profile. This phenomenon is a direct consequence of particle dispersion in the lateral direction, and is seen to be reasonably represented by the present numerical predictions.

Even though underpredicted for low Stokes number particles, the effect of the continuous phase turbulence upon the calculated particle dispersion may be particularly important and even dominant. This is apparent through the particle trajectories represented in Figure 10, where calculations from a ballistic description (where the influence of the fluid turbulence on particle dispersion is simply not considered) are compared with those resulting from inclusion of dispersion for the same particle diameter $\left(d^{\mathrm{p}}=135 \mu \mathrm{m}\right)$ and also for a lower particle 

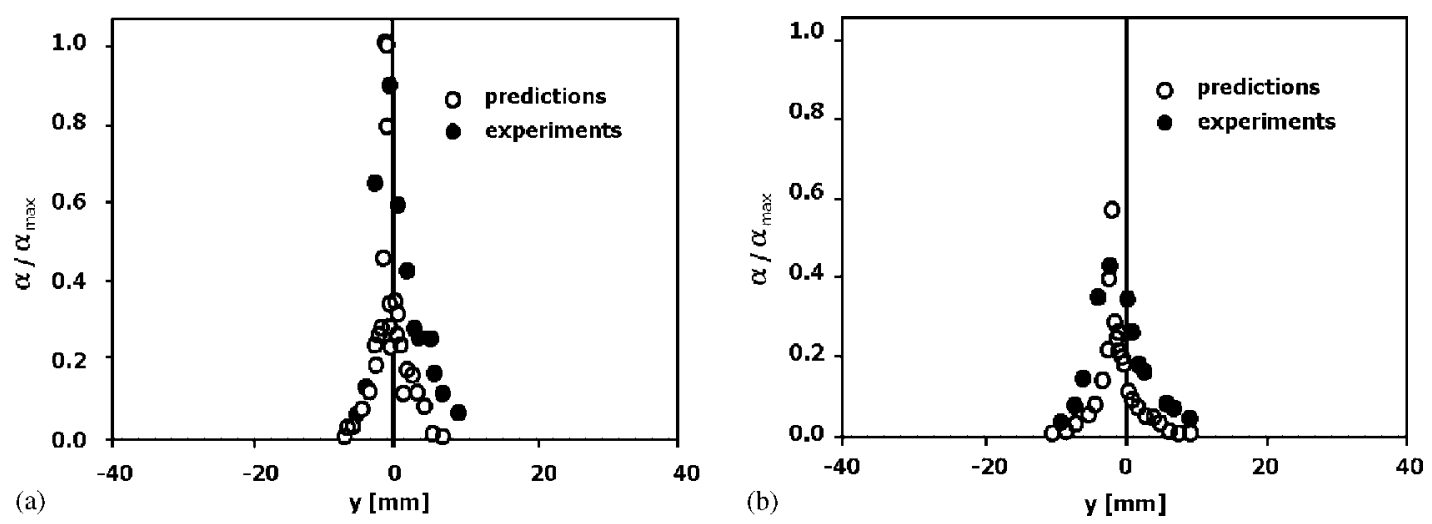

Figure 9. Particle phase, $d^{\mathrm{p}}=135 \mu \mathrm{m}$ : comparison between predicted and measured distributions of normalized particle volume concentration. (a) $x=100 \mathrm{~mm}$; (b) $x=200 \mathrm{~mm}$.

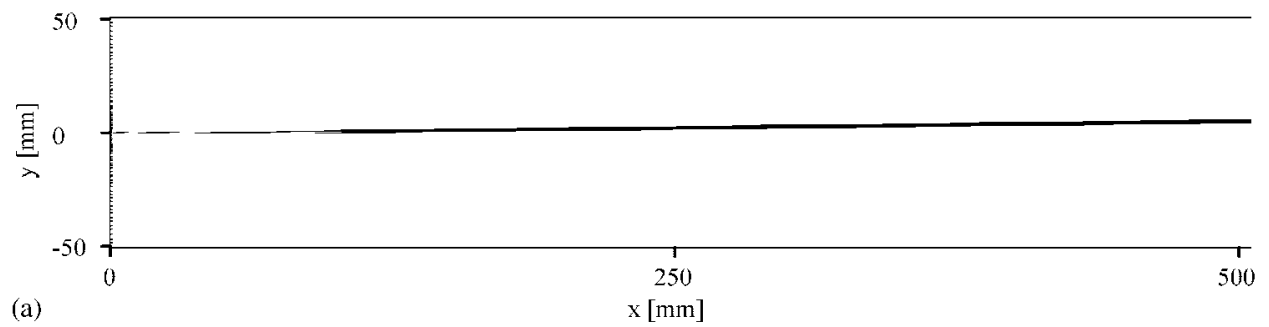

(a)

$\mathrm{x}[\mathrm{mm}]$

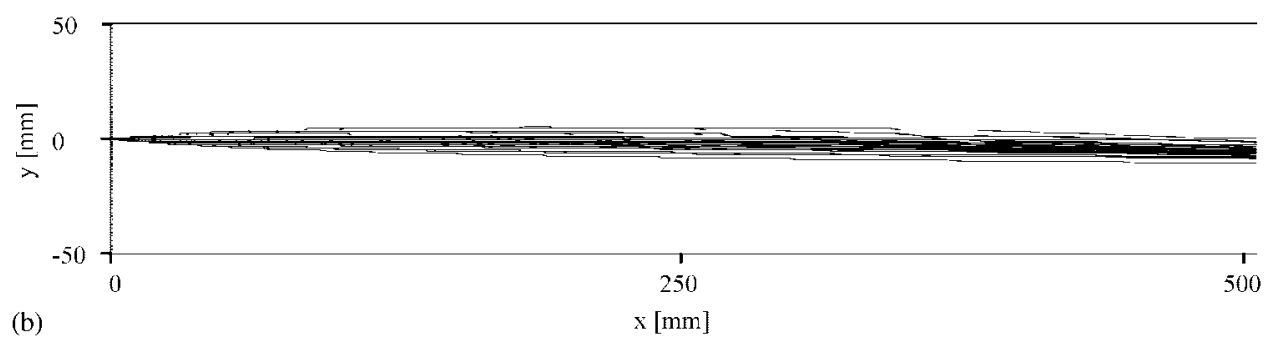

(b)

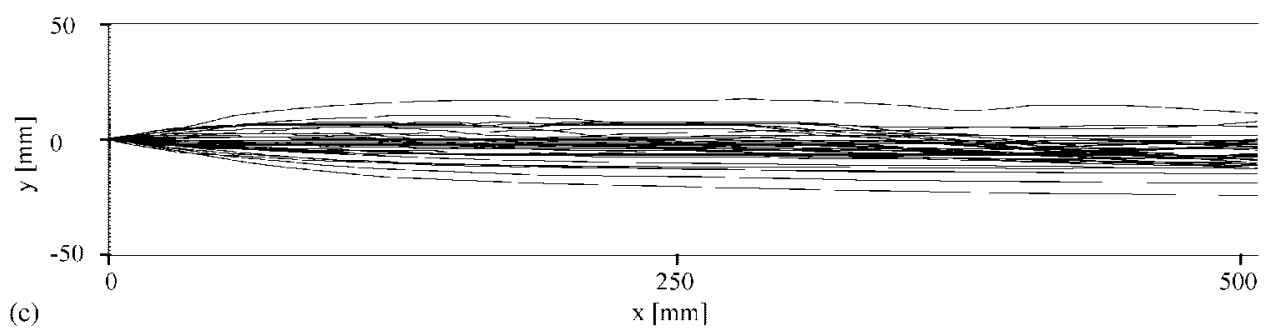

Figure 10. Particle trajectories. (a) $d^{\mathrm{p}}=135 \mu \mathrm{m}$, ballistic approach (turbulent dispersion neglected); (b) $d^{\mathrm{p}}=135 \mu \mathrm{m}$, particle dispersion included; (c) $d^{\mathrm{p}}=42 \mu \mathrm{m}$, particle dispersion included. Only 50 particle starting locations were used for this figure. 
dimension $\left(d^{\mathrm{p}}=42 \mu \mathrm{m}\right)$. The lower effect of particle inertia is clearly visible in this latter case.

In short, single-phase flow phenomena and average particle properties are reasonably well predicted by the present calculation procedure. However, the differences observed between predicted and measured profiles of particle velocity fluctuation (particularly for the smallest Stokes number values), together with the limitations in representing 'overshooting' dispersion (for Stokes numbers of the order of one) suggest that a more elaborate representation of the turbulent transport in both phases would improve the (deliberately simple and easy to implement) basic method described in Section 2.

\section{CONCLUSIONS}

A Lagrangian-Eulerian model for the dispersion of solid particles, in laminar or turbulent, twodimensional flow conditions, using a geometrically versatile CVFEM for the prediction of the continuous phase was reported and tested. Validation tests were performed on the dispersion of particles in a plane mixing layer, a problem for which detailed and reliable measurements are available in the literature. Single-phase flow predictions are in very good agreement with experiments. For two-phase, turbulent flow conditions, the particle statistics were represented through a very simple and easy-to-implement stochastic approach that proved to be rather efficient in predicting the average particle velocity field in both the longitudinal and transverse directions. As expected, owing to the simplistic nature of the turbulence models used in this introductory approach, small particle long-time dispersions, particularly in the lateral direction, tend to be increasingly underpredicted along each particle trajectory. A more elaborate model for particle dispersion, including both spatial and temporal turbulent correlations, should be expected to improve the theoretical predictions. This can be easily implemented without affecting the whole structure of the calculation procedure. On the other hand, a more realistic representation of the time-dependent, large-eddy effect inherent in the fluid shear flow would certainly provide an improvement over the predictive capability of the presently used, isotropic $k-\varepsilon$ type approach for turbulence in the continuous phase. Such improvements and a three-dimensional implementation of the proposed methodology are suggested as useful extensions of the work reported in this paper.

\section{ACKNOWLEDGEMENTS}

This work was conducted under a research project No. PECS/C/SAU/206/95-sponsored by 'Fundação para a Ciência e a Tecnologia' (FCT).

\section{REFERENCES}

1. Crowe CT, Sommerfeld M, Tsuji Y. Multiphase Flows with Droplets and Particles. CRC Press LLC: Boca Raton, FL, 1998.

2. Fan L-S, Zhu C. Principles of Gas-Solid Flows. Cambridge University Press: Cambridge, UK, 1998.

3. Oliveira LA, Costa VF, Baliga BR. A Lagrangian model for the dispersion of solid particles, in two-dimensional flow, using a CVFEM for the prediction of the continuous phase-Computational Mechanics for the Next Millennium, Solid Mechanics and Fluid Mechanics, vol. 1. Pergamon, Elsevier: Amsterdam, 1999; 583-588 (Proceedings of the 4th Asia-Pacific Conference on Computational Mechanics for the Next Millennium (APCOM '99), Singapore, December 15-17, 1999). 
4. Gosman AD, Ionnides E. Aspects of computer simulation of liquid fuelled combustors. AIAA Paper, No. 81-0323, 1981.

5. Crowe CT, Sharma MP, Stock DE. The Particle-Source-In Cell (PSI-CELL) model for gas-droplet flows. Journal of Fluids Engineering 1977; 99:325-332.

6. Ando A, Sadata H, Hishida K, Maeda M. Particle motion in a two-dimensional turbulent mixing layer. Transactions of the Japan Society of Mechanical Engineering B 1990; 56:2189-2197.

7. Hishida K, Ando A, Maeda M. Experiments on particle dispersion in a turbulent mixing layer. International Journal of Multiphase Flow 1992; 18:181-194.

8. Ishima T, Hishida K, Maeda M. Effect of particle residence time on particle dispersion in a plane mixing layer. Journal of Fluids Engineering 1993; 115:751-759.

9. Sommerfeld M (ed.). Proceedings of the 6th Workshop on Two-Phase Flow Predictions. Erlagen, March 30-April 2, 1992.

10. Coimbra CFM, Shirolkar JS, Queiroz McQuay M. Modeling particle dispersion in a turbulent, multiphase mixing layer. Journal of Wind Engineering and Industrial Aerodynamics 1998; 73:79-97.

11. Costa VF, Oliveira LA. A control volume based finite element method for two-dimensional steady fluid flow, heat transfer and related phenomena. Proceedings of the III Encontro Mecânica Computacional, vol. 2. University of Coimbra; Portugal, 1992, p. F10.1.

12. Costa VF, Oliveira LA, Figueiredo AR. A control volume based finite element method for three-dimensional incompressible turbulent fluid flow, heat transfer, and related phenomena. International Journal for Numerical Methods in Fluids 1995; 21:591-613.

13. Launder BE, Spalding DB. Mathematical Models of Turbulence. Academic Press: London, UK, 1972.

14. Costa VF. Modelação Numérica de Escoamentos Tridimensionais Incompressíveis através de um Método de Elementos Finitos Baseado em Volumes de Controlo. Doctoral (Ph.D.) Thesis, University of Coimbra, Portugal, 1995.

15. Patankar SV. Numerical Heat Transfer and Fluid Flow. Hemisphere Publishing Corporation. Washington, DC, 1980.

16. Baliga BR. Control-volume finite element methods for fluid flow and heat transfer. In Advances in Numerical Heat Transfer, Minkowycz WJ, Sparrow EM (eds), Chapter 3, vol. 1. Taylor \& Francis: Washington, DC, USA, 1997.

17. Schneider GE, Raw MJ. A skewed positive influence coefficient upwind procedure for control-volume-based finite element convection-diffusion computation. Numerical Heat Transfer 1986; 9:1-26.

18. Masson C, Saabas HJ, Baliga BR. Co-located equal-order control-volume finite element method for twodimensional axisymmetric incompressible fluid flow. International Journal for Numerical Methods in Fluids 1994; 18:1-26.

19. Maxey MR, Riley JJ. Equation of motion of a small rigid sphere in a nonuniform flow. Physics of Fluids 1983; 26:883-889.

20. Wallis GB. One Dimensional Two-Phase Flow. McGraw-Hill: London, UK, 1969.

21. Oliveira LA. Modelação Numérica da Dispersão de Partículas Sólidas no Escoamento de um Fluido: Conjugação de uma Estratégia Lagrangeana com o Método dos Elementos Finitos Baseado em Volumes de Controlo. Proceedings of the Eventos do Centro Internacional de Matemática (CIM), vol. 6, September 1998 (Escola de Elementos Finitos e Aplicações. University of Coimbra, Portugal, 1998, September 22-October 2 (paper no. $5,1998)$.

22. Chapra SC, Canale RP. Numerical Methods for Engineers. McGraw-Hill International Editions: New York, 1990.

23. Durst F, Milojevic D, Schönung B. Eulerian and Lagrangian predictions of particulate two-phase flows: a numerical study. Applied Mathematical Modelling 1984; 8:101-115.

24. Gouesbet G, Desjonqueres P, Berlemont A. Eulerian and Lagrangian approaches to turbulent dispersion of particles. International Seminar on Transient Phenomenon in Multiphase, ICHMT, Dubrovnik, 1987.

25. Oster D, Wygnanski I. The forced mixing layer between parallel streams. Journal of Fluid Mechanics 1982; 123:91-130. 